\title{
20 years of rituximab treatment: what have we learnt?
}

\author{
Christoph Renner*,1 \\ ${ }^{1}$ OnkoZentrum Hirslanden, Zürich, Switzerland \\ *Author for correspondence: Christoph.Renner@hirslanden.ch
}

\begin{abstract}
"Rituximab represents one of the first mAbs for which biosimilars have become available and have entered clinical practice. This might allow for increased access, particularly in healthcare systems where resources are limited."
\end{abstract}

First draft submitted: 30 October 2019; Accepted for publication: 30 October 2019; Published online: 13 November 2019

Keywords: B cell • biosimilar • immunotherapy • lymphoma • monoclonal antibody • rituximab

Rituximab, initially approved in 1997 by the US FDA for use in relapsed/refractory indolent non-Hodgkin lymphoma (NHL) and subsequently in other indications, was the first monoclonal antibody $(\mathrm{mAb})$ utilized in oncology and remains widely used over two decades later [1]. The introduction of rituximab revolutionized the treatment of B-cell lymphomas and was an important milestone in the advent of newer immunotherapeutic approaches. Although research into immunotherapy spans more than a century, the development of rituximab was made possible through production technologies established in 1975, when Köhler and Milstein developed the first hybridoma cell lines capable of producing large quantities of mAbs for virtually any antigen of interest, thereby paving the way for the first use of mAbs in a human application in $1986[2,3]$.

Rituximab is a chimeric mouse/human $\mathrm{mAb}$ that binds with high affinity to $\mathrm{CD} 20$, a glycosylated transmembrane protein that is expressed on the surface of most malignant cells and on normal B cells during development from pre-B cells to mature B cells. CD20 is not known to be expressed on B cell progenitors or mature plasma cells, or in other normal tissues of the body $[2,4,5]$. In Phase I and II studies, rituximab demonstrated potent B-celldepleting activity in peripheral blood and lymphoid tissues [6,7]. Antibody-dependent cell-mediated cytotoxicity and complement-dependent cytotoxicity are among the key mechanisms contributing to rituximab-mediated B-cell depletion.

During its development and subsequent use, several obstacles to the use of rituximab have been raised. The potential effectiveness and tolerability of any $\mathrm{mAb}$ that destroys not only tumor cells, but also physiological cells, provoked skepticism initially. However, rituximab has demonstrated an impressive efficacy and safety profile both as monotherapy (induction and/or maintenance) and in combination with chemotherapy, in the treatment of patients with various CD20-expressing lymphoid malignancies. Importantly, the elimination of normal B cells was found to be tolerated by most patients [1].

Due to its chimeric nature and the expression of the CD20 target antigen on physiological B cells, it was initially assumed that only a short duration of therapy would be possible with rituximab, although this has proven not to be the case. Rituximab maintenance therapy is indicated in Europe for the treatment of previously untreated or relapsed/refractory follicular lymphoma (FL) in patients responding to induction therapy, and is recommended in the European Society of Medical Oncology clinical practice guidelines for the treatment of high tumor burden Stage III/IV FL, for up to 2 years [8].

Evidence shows that rituximab maintenance therapy, after induction treatment with rituximab alone or chemotherapy with or without rituximab, is associated with improvement in progression-free survival (PFS) in both treatment-naïve and previously treated patients with FL [9]. In the Phase III Primary RItuximab and MAintenance (PRIMA) trial, 2 years of rituximab maintenance therapy after immunochemotherapy with rituximab as first-line treatment significantly improved PFS in patients with previously untreated high tumor burden FL (3-year PFS rate: $74.9 \%$ for rituximab maintenance therapy vs $57.6 \%$ for observation only; hazard ratio [HR]: $0.55 ; \mathrm{p}<0.001)$ [10].

Future $\because$ Medicine 
Long-term follow-up (median of 73 months) showed that this PFS benefit was maintained $(59.2 \%$ vs $42.7 \%$; HR: 0.58; p < 0.0001) [11]. In the Phase III SAKK 35/03 trial in patients with untreated, relapsed, stable or chemotherapy-resistant FL, long-term rituximab maintenance therapy for a maximum of 5 years doubled the median PFS compared with short-term therapy for 8 months ( 7.4 vs 3.5 years, respectively; $p=0.04$ ), although long-term treatment was associated with increased toxicity [12].

Historically, immunotherapy and chemotherapy were considered to be antagonistic treatments [13]. It was assumed that intensive chemotherapy would suppress an immune response in patients with aggressive lymphomas, and therefore combining these agents with rituximab would make little sense. To the contrary, there is a strong rationale for this combination, based on the distinct mechanisms of action of these agents and the evidence that rituximab may sensitize chemoresistant tumor cells to the actions of cytotoxic drugs [14]. This has been borne out by the success of treatment with rituximab in combination with chemotherapy regimens in multiple studies. In an initial study in patients with low-grade NHL $(\mathrm{n}=35)$, rituximab added to cyclophosphamide, doxorubicin, vincristine and prednisone (R-CHOP) resulted in an overall response rate of $100 \%$, and $63 \%$ of patients achieved a complete response, with no significant increase in toxicity [14].

The benefits of combining rituximab with CHOP have also been demonstrated in patients with aggressive lymphomas. The LNH-98.5 trial conducted by the Groupe d'Etudes des Lymphomes de l'Adulte (GELA) was the first randomized study comparing R-CHOP with a standard CHOP regimen in previously untreated elderly patients with diffuse large B-cell lymphoma (DLBCL, $\mathrm{n}=399 ; 60 \%$ poor risk). Compared with CHOP, the combination regimen was associated with a significantly greater proportion of complete responders $(76 \%$ vs $63 \%$, respectively; $\mathrm{p}=0.005)$. In addition, at 2 years, longer event-free survival $(57 \%$ vs $38 \%$; HR: $0.58 ; \mathrm{p}<0.001)$ and OS (70\% vs 57\%; HR: $0.64 ; \mathrm{p}=0.007)$ were observed [15]. Results from a 10-year analysis have confirmed the benefits of R-CHOP in this patient population [16]. Evidence also shows that approximately $50-70 \%$ of patients with DLBCL can achieve complete cure with R-CHOP.

It might be hypothesized that the potent immunosuppression and B-cell depletion associated with rituximab therapy would predispose patients to infections, which is a particular concern in patients with cancer. Reassuringly, although rituximab may lead to a decrease in immunoglobulin levels and a small increase in infections overall [17], severe infections occur less frequently than expected, and B cell recovery occurs around 6-12 months after discontinuing therapy $[18,19]$. Indeed, a systematic review and meta-analysis of 17 randomized controlled trials in adult patients with CD20-positive malignant lymphomas $(n=5259)$ found that the addition of rituximab to standard chemotherapy did not increase the overall incidence of severe infections compared with standard chemotherapy alone [19]. Concerns have also been raised over whether the production of human anti-chimeric antibodies (HACA) to the murine fragments of rituximab may lead to loss of efficacy with repeated use of the drug [20]. Although high HACA titers have been reported with rituximab treatment, these are most commonly seen in patients with autoimmune conditions [20], and may be less of a concern in hematologic malignancies.

We now have over 20 years of clinical experience with rituximab. Despite early skepticism, and some remaining unknowns, rituximab has demonstrated a convincing efficacy and safety profile, improving outcomes remarkably in B-cell malignancies including FL, DLBCL and chronic lymphocytic leukaemia. Following its introduction, rituximab was rapidly accepted within the field, and remains well-established as one of the most widely used agents in current treatment regimens for B-cell lymphomas. Building on the success of early antibody therapy, rituximab has also played a pivotal role in supporting the development of a plethora of newer immunotherapies.

Clearly, given the importance of rituximab in lymphoma treatment strategies, widespread access to this therapy is essential. However, this is not the case in all countries, meaning some patients may be receiving suboptimal treatment. Rituximab represents one of the first $\mathrm{mAbs}$ for which biosimilars have become available and have entered clinical practice. This might allow for increased access, particularly in healthcare systems where resources are limited.

The article by Jurczak et al. in this issue provides a concise summary of the evidence for one rituximab biosimilar (Sandoz rituximab) compared with reference rituximab [21].

Financial \& competing interests disclosure

C Renner has received travel support and/or honoraria from Roche, Celgene, Janssen, Gilead, Sandoz and Takeda. The author has no other relevant affiliations or financial involvement with any organization or entity with a financial interest in or financial conflict with the subject matter or materials discussed in the manuscript apart from those disclosed. 
Editorial support for the development of this article was provided by Ben Caldwell of Spirit, a division of the Spirit Medical Communications Group, Manchester, UK, and funded by Sandoz. The views and opinions expressed in this article are those of the author alone.

\section{Open access}

This work is licensed under the Attribution-NonCommercial-NoDerivatives 4.0 Unported License. To view a copy of this license, visit http://creativecommons.org/licenses/by-nc-nd/4.0/

\section{References}

1. Salles G, Barrett M, Foà R et al. Rituximab in B-cell hematologic malignancies: a review of 20 years of clinical experience. Adv. Ther. 34(10), 2232-2273 (2017).

2. Pierpont TM, Limper CB, Richards KL. Past, present, and future of rituximab-the world's first oncology monoclonal antibody therapy. Front. Oncol. 8, 163 (2018).

3. Snook AE, Waldman SA. Advances in cancer immunotherapy. Discov. Med. 15(81), 120-125 (2013).

4. Maloney DG. Preclinical and Phase I and II trials of rituximab. Semin. Oncol. 26(5 Suppl. 14), 74-78 (1999).

5. Leandro MJ. B-cell subpopulations in humans and their differential susceptibility to depletion with anti-CD20 monoclonal antibodies. Arthritis Res. Ther. 15(Suppl. 1), S3 (2013).

6. Maloney DG, Liles TM, Czerwinski DK et al. Phase I clinical trial using escalating single-dose infusion of chimeric anti-CD20 monoclonal antibody (IDEC-C2B8) in patients with recurrent B-cell lymphoma. Blood 84(8), 2457-2466 (1994).

7. Maloney DG, Grillo-Lopez AJ, White CA et al. IDEC-C2B8 (rituximab) anti-CD20 monoclonal antibody therapy in patients with relapsed low-grade non-Hodgkin's lymphoma. Blood 90(6), 2188-2195 (1997).

8. Dreyling M, Ghielmini M, Rule $S$ et al. Newly diagnosed and relapsed follicular lymphoma: ESMO clinical practice guidelines for diagnosis, treatment and follow-up. Ann. Oncol. 27(Suppl. 5), v83-v90 (2016).

9. Fowler NH. Role of maintenance rituximab (rituxan) therapy in the treatment of follicular lymphoma. P T 36(9), 590-598 (2011).

10. Salles G, Seymour JF, Offner F et al. Rituximab maintenance for 2 years in patients with high tumour burden follicular lymphoma responding to rituximab plus chemotherapy (PRIMA): a Phase III, randomised controlled trial. Lancet 377(9759), 42-51 (2011).

11. Salles G, Seymour JF, Feugier P et al. Updated 6 year follow-up of the PRIMA study confirms the benefit of 2-year rituximab maintenance in follicular lymphoma patients responding to frontline immunochemotherapy. Blood 122(21), abstract 509 (2013).

12. Taverna CJ, Martinelli G, Hitz F et al. Rituximab maintenance treatment for a maximum of 5 years in follicular lymphoma: results of the randomized Phase III trial SAKK 35/03. Blood 122(21), abstract 508 (2013).

13. McCoy MJ, Nowak AK, Lake RA. Chemoimmunotherapy: an emerging strategy for the treatment of malignant mesothelioma. Tissue Antigens 74(1), 1-10 (2009).

14. Czuczman MS. Combination chemotherapy and rituximab. Anticancer Drugs 12(Suppl. 2), S15-S19 (2001).

15. Coiffier B, Lepage E, Briere J et al. CHOP chemotherapy plus rituximab compared with $\mathrm{CHOP}$ alone in elderly patients with diffuse large-B-cell lymphoma. N. Engl. J. Med. 346(4), 235-242 (2002).

16. Coiffier B, Thieblemont C, Van Den Neste E et al. Long-term outcome of patients in the LNH-98.5 trial, the first randomized study comparing rituximab-CHOP to standard CHOP chemotherapy in DLBCL patients: a study by the Groupe d'Etudes des Lymphomes de l'Adulte. Blood 116(12), 2040-2045 (2010).

17. Vidal L, Gafter-Gvili A, Salles G et al. Rituximab maintenance improves overall survival of patients with follicular lymphoma-individual patient data meta-analysis. Eur. J. Cancer 76, 216-225 (2017).

18. Kimby E. Tolerability and safety of rituximab (MabThera). Cancer Treat. Rev. 31(6), 456-473 (2005).

19. Lanini S, Molloy AC, Fine PE, Prentice AG, Ippolito G, Kibbler CC. Risk of infection in patients with lymphoma receiving rituximab: systematic review and meta-analysis. BMC Med. 9, 36 (2011).

20. Lunardon L, Payne AS. Inhibitory human antichimeric antibodies to rituximab in a patient with pemphigus. J. Allergy Clin. Immunol. 130(3), 800-803 (2012).

21. Jurczak W, Cohen S, Illidge T, Da Silva A, Amersdorffer J. Scientific rationale underpinning development of biosimilar rituximab in hematological cancers and inflammatory diseases. Fut. Oncol. doi:10.2217/fon-2019-0430 (2019) (Epub ahead of print). 
